\title{
Inhibition of Streptococcus pyogenes growth by native Australian plants: New approaches towards the management of impetigo, pharyngitis and rheumatic heart disease
}

\author{
Cameron Jay Lee ${ }^{1}$, Mitchell Henry Wright ${ }^{1}$, Megan Sarah Jean Arnold ${ }^{1,2}$, Anthony Carlson Greene ${ }^{1}$, Ian Edwin Cock ${ }^{1,3^{*}}$ \\ 'School of Natural Sciences, Nathan Campus, Griffith University, 170 Kessels Rd, Nathan, Queensland 4111, AUSTRALIA. \\ 2Eskitis Institute for Drug Discovery, Griffith University, Queensland 4111, AUSTRALIA. \\ 'Environmental Futures Research Institute, Nathan Campus, Griffith University, 170 Kessels Rd, Nathan, Queensland 4111, AUSTRALIA.
}

\begin{abstract}
Introduction: Streptococcus pyogenes can cause a variety of diseases including streptococcal pharyngitis, impetigo and rheumatic heart disease, dependant on which tissue it infects. Many Australian plants have documented therapeutic properties as general antiseptics, but are yet to be tested for the ability to inhibit $S$. pyogenes growth. Methods: Solvent extracts were prepared using Australian plants with documented ethnobotanical usage to treat bacterial infections, or published antibacterial activity. The extracts were investigated by disc diffusion assay for the ability to inhibit the growth of a clinical strain of $S$. pyogenes. Their MIC values were determined to quantify and compare their efficacies. Toxicity was determined using the Artemia franciscana nauplii bioassay. Results: $S$. pyogenes growth was inhibited by 24 of the 34 extracts tested. The Eucalyptus spp. extracts were particularly potent. MIC values of 341 and $88 \mu \mathrm{g} /$ $\mathrm{mL}$ were determined for the aqueous and methanolic $E$. baileyana extracts respectively. Similarly, MIC values of 134 and $53 \mu \mathrm{g} / \mathrm{mL}$ were determined for the aqueous and methanolic E. major extracts respectively. The methanolic wattle seed extract, aqueous and methanolic lemon aspen extracts, aqueous native thyme extract, methanolic river mint extract and the methanolic native basil extract were similarly potent growth inhibitors, with MIC values $\leq 1000 \mu \mathrm{g} / \mathrm{mL}$. Several other extracts (methanolic native tamarind, bush tomato, desert lime, native thyme, native sage and the T. stipitata leaf extracts, as well as the aqueous river mint, native basil, T. stipitata leaf extracts) displayed moderate growth inhibitory activity (MIC $=1000-5000$ $\mu \mathrm{g} / \mathrm{mL}$ ). All other extracts were either low potency S. pyogenes growth inhibitors or were devoid of inhibitory activity. The E. baileyana and E. major
\end{abstract}

methanolic extracts, as well as the $E$. baileyana aqueous extract induced significant mortality in the Artemia fransiscana bioassay, with $\mathrm{LC}_{50}$ values substantially $<1000 \mu \mathrm{g} / \mathrm{mL}$. All other extracts were nontoxic, with $\mathrm{LC}_{50}$ values $>1000 \mu \mathrm{g} / \mathrm{mL}$. Conclusion: The potent growth inhibitory bioactivity of the Eucalyptus spp., lemon aspen, wattle seed, native basil and river mint extracts against $S$. pyogenes demonstrates their potential for the treatment and prevention of $S$. pyogenes induced disease. However, the toxicity of the Eucalyptus spp. extracts may limit their use to topical treatments for pharyngitis and impetigo. As the lemon aspen, wattle seed, native basil and river mint extracts were nontoxic, they may also have wider uses in treating systemic illnesses such as rheumatic fever, rheumatic heart disease and cellulitis.

Key words: Eucalyptus, Antioxidant, Lemon aspen, Pharyngitis, Impetigo, Rheumatic heart disease, Antibacterial activity.

\section{Correspondence:}

Ian Edwin Cock

School of Natural Sciences,

Nathan Campus, Griffith University, 170 Kessels Rd, Nathan,

Queensland 4111, AUSTRALIA.

Tel no: +61 737357637

E-mail: I.Cock@griffith.edu.au

DOI : 10.5530/pc.2016.3.6

\section{INTRODUCTION}

Streptococcus pyogenes is a gram-positive, facultative anaerobe and the etiological agent of a number of diseases in humans. In nature, the bacterium is present as part of the natural skin flora of humans and animals which under certain conditions can cause disease. ${ }^{1}$ Associated diseases vary in symptoms and severity, ranging from superficial skin infections (impetigo and ecthyma) to rheumatic fever. ${ }^{2,3}$ Typically, streptococcal infections are localised on the epidermis or nasopharynx and/or oropharynx and are treated with antibiotics as required. However, due to the selflimiting nature of these infections and the increasing risk of antibiotic resistance unless complications arise monitoring and symptom treatment is often the preferred option. The probing of natural plant resources offers an alternate means of fighting streptococcal diseases through the prevention of bacterial growth. ${ }^{4}$

Several Australian plant species were selected for S. pyogenes growth inhibitory activity screening based on their usage in traditional medicine systems ${ }^{5}$ and/or their reported antibacterial activities ${ }^{6-9}$ (Table 1). Plants of the genus Eucalyptus are particularly well known for their antiseptic properties due to their high 1, 8-cineole contents. ${ }^{5,10-12}$ The first Australians crushed the leaves and inhaled the volatiles to treat coughs and colds. ${ }^{5,10}$ Fresh leaves or decoctions prepared from the leaves were also used as wound antiseptics and to treat skin and throat infections. Furthermore, in vitro studies have demonstrated the growth inhibitory properties of Eucalyptus baileyana and Eucalyptus major extracts towards a panel of bacterial species, indicating their therapeutic potential in treating pathogenic diseases. ${ }^{11,12}$ Indeed, essential oils prepared from Eucalyptus spp. leaves remain a popular antiseptic agent, not only in Australia, but are also commonly sold in pharmacies internationally.

Whilst the ethnobotanical uses of many Australian plants in traditional Aboriginal medicine systems have been recorded, rigourous scientific studies are lacking for many species. ${ }^{5,10}$ Recent studies have reported potent broad spectrum bacterial growth inhibitory activity for S. spinescens extracts. ${ }^{13}$ Despite this, $S$. spinescens is yet to be evaluated for S. pyogenes growth inhibitory activity. Tasmannia lanceolata ${ }^{14}$ and Tasmannia stipitata (family Winteraceae) extracts have potent broad spectrum antimicrobial activity for in vitro. ${ }^{6}$ Furthermore, T. lanceolata ${ }^{15}$ and T. stipitata ${ }^{6}$ have also been reported to inhibit the proliferation of the gastrointestinal protozoal parasite Giardia duodenalis. ${ }^{6,15}$ We were unable to find similar studies examining the therapeutic potential of T. insipidia.

Recent studies have reported exceptionally high antioxidant content of the fruits of several Australian plant species. ${ }^{8,916}$ In particular, these 
studies reported the fruit of Kunzea pomifera F. Muell. (muntries) and Podocarpus elatus R. Br. (Illawarra plum) to have similar antioxidant capacities to blueberries (which are themselves considered to have a high antioxidant capacity). Acronychia acidula F. Muell. (lemon aspen), Citrus glauca (Lindl.) Burkill (desert lime) and Solanum aviculare G. Forst. (bush tomato) have also been reported to have high antioxidant capacities. ${ }^{9,16}$ It has been postulated that the high antioxidant contents of some Australian native fruits may provide them with therapeutic effects. ${ }^{14,16-20}$ Similarly, a number of Australian culinary herbs including Prostanthera rotundifolia $\mathrm{R}$. Br. (native thyme), Prostanthera incise R. $\mathrm{Br}$. (native sage), and Mentha australis R. Br. (rivermint) have been reported to have high antioxidant capacities and potent growth inhibitory activity against bacteria associated with the induction of several autoimmune inflammatory diseases. ${ }^{20}$ Despite this relative wealth of information documenting antibacterial Australian plants, many are yet to be tested for the ability to inhibit $S$. pyogenes growth. The current study examines the growth inhibitory activity of extracts of selected Australian plants against $S$. pyogenes, and thus their potential in the prevention and treatment of streptococcal pharyngitis, impetigo, rheumatic fever and rheumatic heart disease.

\section{MATERIALS AND METHODS}

\section{Plant source and extraction}

Acronychia acidula F. Muell. (lemon aspen), Podocarpus elatus R. Br. (Illawarra plum), Kunzea pomifera F. Muell. (muntries), Diploglottis australis Hook. f. (native tamarind), Acacia vivtoriae Benth. (wattle seed), Citrus glauca (Lindl.) Burkill (desert lime), Solanum aviculare G. Forst. (bush tomato), Prostanthera incise R. Br. (native sage), Prostanthera rotundifolia R. Br. (native thyme), Ocimum tenuiflorum L. (native basil) and Mentha australis R. Br. (river mint) were obtained from Taste of Australia Bush Food, Australia. Air dried Tasmannia insipidia R. Br. leaves and Tasmannia stipitata (Vick.) A. C. Smith leaves and berries were supplied and verified by the Queensland Bush foods Association, Australia. Scaevola spinescens R. Br. was supplied by Jeannie Crago of Outback Books Australia (a commercial supplier of S. spinescens tea) as a pre-dried and course milled whole plant material. Eucalyptus baileyana F. Muell. And Eucalyptus major (Maiden) Blakely plant materials were collected from Toohey Forest, Brisbane and were identified with reference to a taxonomic key to Toohey Forest plants. ${ }^{24}$ Voucher samples of all plant specimens are stored in the School of Natural Sciences, Griffith University (Australia). The plant materials were comprehensively dried in a Sunbeam food dehydrator and the dried plant materials were stored at $-30^{\circ} \mathrm{C}$. Prior to use, the plant materials were thawed and freshly ground to a coarse powder. Individual $1 \mathrm{~g}$ quantities of the ground plant material were weighed into separate tubes and $50 \mathrm{~mL}$ of water or methanol were added. All solvents were obtained from Ajax and were AR grade. The ground plant materials were individually extracted in each solvent for $24 \mathrm{~h}$ at $4^{\circ} \mathrm{C}$ with gentle shaking. The extracts were subsequently filtered through filter paper (Whatman No. 54) under vacuum, followed by drying by rotary evaporation in an Eppendorf concentrator 5301. The resultant dry extract was weighed and redissolved in $10 \mathrm{~mL}$ deionised water (containing 1\% DMSO).

\section{Qualitative phytochemical studies}

Phytochemical analysis of the extracts for the presence of triterpenoids, cardiac glycosides, flavonoids, saponins, phenolic compounds, phytosteroids, anthraquinones, tannins and alkaloids was conducted by previously described assays. ${ }^{25-27}$

\section{Antibacterial screening}

\section{Clinical Streptococcus pyogenes strain}

The clinical isolate strain of Streptococcus pyogenes used in this study was supplied by Ms. Michelle Mendell of the School of Natural Sciences Griffith University, Australia. All growth studies were performed using nutrient agar (Oxoid Ltd., Australia) under aerobic conditions. Incubation was at $30^{\circ} \mathrm{C}$ and the stock culture was subcultured and maintained in nutrient broth at $4^{\circ} \mathrm{C}$.

\section{Evaluation of antimicrobial activity}

Antimicrobial activity of all plant extracts was determined using a modified disc diffusion assay. ${ }^{28,29}$ Briefly, $100 \mu \mathrm{L}$ of $S$. Pyogenes culture was grown in $10 \mathrm{~mL}$ of fresh nutrient broth until they reached a count of $\sim 10^{8}$ cells $/ \mathrm{mL}$. A volume of $100 \mu \mathrm{L}$ of the bacterial suspension was spread onto nutrient agar plates and extracts were tested for antibacterial activity using $5 \mathrm{~mm}$ sterilised filter paper discs. Discs were infused with 10 $\mu \mathrm{L}$ of the plant extracts, allowed to dry and placed onto the inoculated plates. The plates were allowed to stand at $4^{\circ} \mathrm{C}$ for $2 \mathrm{~h}$ before incubation at $30^{\circ} \mathrm{C}$ for $24 \mathrm{~h}$. The diameters of the inhibition zones were measured to the closest whole millimetre. Each assay was performed in at least triplicate. Mean values $( \pm$ SEM) are reported in this study. Standard discs of ampicillin $(10 \mu \mathrm{g})$ were obtained from Oxoid, Australia and were used as positive controls to compare antibacterial activity. Filter discs infused with $10 \mu \mathrm{L}$ of distilled water were used as a negative control.

\section{Minimum inhibitory concentration (MIC) determination}

The minimum inhibitory concentration (MIC) of each extract against $S$. pyogenes was determined as previously described. ${ }^{28,30}$ Briefly, the plant extracts were diluted in deionised water and tested across a range of concentrations. Discs were infused with $10 \mu \mathrm{L}$ of the test dilutions, allowed to dry and placed onto inoculated plates. The assay was completed as outlined above and graphs of the zone of inhibition versus concentration were plotted for each extract. Linear regression was used to determine the MIC values of each extract.

\section{Toxicity screening}

\section{Reference toxin for toxicity screening}

Potassium dichromate $\left(\mathrm{K}_{2} \mathrm{Cr}_{2} \mathrm{O}_{7}\right)$ (AR grade, Chem-Supply, Australia) was prepared as a $4 \mathrm{mg} / \mathrm{mL}$ solution in distilled water and was serially diluted in artificial seawater for use in the Artemia franciscana nauplii bioassay.

\section{Artemia franciscana nauplii toxicity screening}

Toxicity was tested using an adapted Artemia franciscana nauplii lethality assay as previously described. ${ }^{31-33}$ Briefly, $400 \mu \mathrm{L}$ of seawater containing approximately 43 (mean 43.2, $\mathrm{n}=155$, SD 14.5) A. franciscana nauplii were added to wells of a 48 well plate and immediately used for bioassay. A volume of $400 \mu \mathrm{L}$ of diluted plant extracts or the reference toxin were transferred to the wells and incubated at $25 \pm 1^{\circ} \mathrm{C}$ under artificial light (1000 Lux). A $400 \mu \mathrm{L}$ seawater negative control was run in triplicate for each plate. All treatments were performed in at least triplicate. The wells were checked at regular intervals and the number of dead counted. The nauplii were considered dead if no movement of the appendages was detected within $10 \mathrm{sec}$. After $24 \mathrm{~h}$ all nauplii were sacrificed and counted to determine the total $\%$ mortality per well. The $\mathrm{LC}_{50}$ with $95 \%$ confidence limits for each treatment was determined using probit analysis.

\section{Statistical analysis}

Data are expressed as the mean \pm SEM of at least three independent experiments. 
Table 1: The medicinal usage, common names and known constituents of the native Australian plant species tested in this study

\begin{tabular}{|c|c|c|c|c|c|}
\hline Plant Species & $\begin{array}{l}\text { Part Used in This } \\
\text { Study }\end{array}$ & Common Name/s & $\begin{array}{c}\text { Traditional Medicinal Uses and Known } \\
\text { Therapeutic Properties }\end{array}$ & Known Constituents & References \\
\hline Acacia vivtoriae Benth. & seed & wattle & $\begin{array}{l}\text { The seed was ground into a flour and } \\
\text { used to make a bread. The seed has } \\
\text { reported antibacterial and anticancer } \\
\text { properties. Other Acacia spp. were used } \\
\text { to treat allergies, rash and as an antiseptic. }\end{array}$ & $\begin{array}{l}\text { Unknown, although } \\
\text { other Acacia spp. contain } \\
\text { high levels of tannins, } \\
\text { terpenoids and saponins }\end{array}$ & 5,10 \\
\hline $\begin{array}{l}\text { Acronychia acidula F. } \\
\text { Muell. }\end{array}$ & fruit & lemon aspen & $\begin{array}{l}\text { The fruit has a high antioxidant capacity } \\
\text { and was mainly used as a nutritious food. } \\
\text { The fruit has reported antibacterial and } \\
\text { anticancer properties. }\end{array}$ & $\begin{array}{l}\text { unknown although } \\
\text { the related species } \\
\text { Acronychia baueri has } \\
\text { been reported to contain } \\
\text { alkaloids }\end{array}$ & 10 \\
\hline $\begin{array}{l}\text { Citrus glauca (Lindl.) } \\
\text { Burkill }\end{array}$ & fruit & desert lime & $\begin{array}{l}\text { The fruit has a high antioxidant capacity } \\
\text { and was mainly used as a nutritious food. } \\
\text { The fruit has reported antibacterial and } \\
\text { anticancer properties. }\end{array}$ & Unknown & $16,19,20$ \\
\hline $\begin{array}{l}\text { Diploglottis australis } \\
\text { Hook. f. }\end{array}$ & fruit & $\begin{array}{l}\text { Australian native } \\
\text { tamarind }\end{array}$ & $\begin{array}{l}\text { The fruit has a high antioxidant capacity } \\
\text { and was mainly used as a nutritious food. } \\
\text { The fruit has reported antibacterial and } \\
\text { anticancer properties. }\end{array}$ & Unknown & $16,19,20$ \\
\hline $\begin{array}{c}\text { Eucalyptus baileyana } \mathrm{F} . \\
\text { Muell. }\end{array}$ & leaf & Bailey's stringybark & $\begin{array}{l}\text { Used to treat rheumatism, swelling, } \\
\text { inflammation, skin disorders stomach } \\
\text { disorders, bactericide (wounds, sores) }\end{array}$ & high terpene content & $5,10,12$ \\
\hline $\begin{array}{l}\text { Eucalyptus major } \\
\text { (Maiden) Blakely }\end{array}$ & leaf & grey gum & $\begin{array}{l}\text { Used to treat rheumatism, swelling, } \\
\text { inflammation, skin disorders stomach } \\
\text { disorders, bactericide (wounds, sores) }\end{array}$ & high terpene content & $5,10,12$ \\
\hline $\begin{array}{l}\text { Kunzea pomifera } \mathrm{F} . \\
\text { Muell. }\end{array}$ & fruit & muntries & $\begin{array}{l}\text { The fruit has a high antioxidant capacity } \\
\text { and was mainly used as a nutritious food. } \\
\text { The fruit has reported antibacterial and } \\
\text { anticancer properties. }\end{array}$ & $\begin{array}{l}\text { ellagitannins } \\
\text { (including ellagic acid), } \\
\text { gallotannins, terpenoids, } \\
\text { purine analogues }\end{array}$ & $5,10,21$ \\
\hline Mentha australis $\mathrm{R} . \mathrm{Br}$. & leaf & river mint & $\begin{array}{l}\text { The leaf has a high antioxidant capacity } \\
\text { and was mainly used as a nutritious food. } \\
\text { The leaf has reported antibacterial and } \\
\text { anticancer properties. }\end{array}$ & $\begin{array}{l}\text { high in essential oils; } \\
\text { constituents have not } \\
\text { been fully established. }\end{array}$ & 10 \\
\hline Ocimum tenuiflorum $\mathrm{L}$. & leaf & $\begin{array}{l}\text { Australian native } \\
\text { basil }\end{array}$ & $\begin{array}{l}\text { The leaf has a high antioxidant capacity } \\
\text { and was mainly used as a nutritious food. } \\
\text { The leaf has reported antibacterial and } \\
\text { anticancer properties. }\end{array}$ & $\begin{array}{l}\text { unknown although the } \\
\text { related species Ocimum } \\
\text { sanctum is rich in } \\
\text { polyphenolic compounds } \\
\text { including eugenol }\end{array}$ & 19 \\
\hline $\begin{array}{c}\text { Podocarpus elatus } \mathrm{R} . \\
\text { Br. }\end{array}$ & fruit & Illawarra plum & $\begin{array}{l}\text { The fruit has a high antioxidant capacity } \\
\text { and was mainly used as a nutritious food. } \\
\text { The fruit has reported antibacterial and } \\
\text { anticancer properties. }\end{array}$ & unknown & $16,19,20$ \\
\hline $\begin{array}{l}\text { Prostanthera incisa } \\
\text { R. Br. }\end{array}$ & leaf & $\begin{array}{l}\text { Australian native } \\
\text { sage }\end{array}$ & $\begin{array}{l}\text { The leaf has a high antioxidant capacity } \\
\text { and was mainly used as a nutritious food. } \\
\text { The leaf has reported antibacterial and } \\
\text { anticancer properties. }\end{array}$ & $\begin{array}{l}\text { high in essential oils; } \\
\text { constituents have not } \\
\text { been fully established. }\end{array}$ & 10 \\
\hline $\begin{array}{l}\text { Prostanthera } \\
\text { rotundifolia } \mathrm{R} . \mathrm{Br} .\end{array}$ & leaf & $\begin{array}{l}\text { Australian native } \\
\text { thyme }\end{array}$ & $\begin{array}{l}\text { The leaf has a high antioxidant capacity } \\
\text { and was mainly used as a nutritious food. } \\
\text { The leaf has reported antibacterial and } \\
\text { anticancer properties. }\end{array}$ & $\begin{array}{l}\text { high in essential oils, } \\
\text { particularly 1,8-cineole }\end{array}$ & 10 \\
\hline $\begin{array}{l}\text { Scaevola spinescens } \\
\text { R. Br. }\end{array}$ & leaf & $\begin{array}{l}\text { currant bush, } \\
\text { maroon bush, } \\
\text { prickly fan flower }\end{array}$ & $\begin{array}{l}\text { Used as an antiseptic (especially for skin } \\
\text { disorders/sores), cancer, pain and urinary } \\
\text { disorders. Antiviral properties have also } \\
\text { recently been reported. }\end{array}$ & $\begin{array}{l}\text { pentacyclic triterpenoids } \\
\text { including lupeol, } \\
\text { taraxerol, myricadiol), } \\
\text { coumarins (including } \\
\text { ammarin, nodakenetin), } \\
\text { scaevoloside }\end{array}$ & $1,10,13,21,22$ \\
\hline
\end{tabular}




\begin{tabular}{|c|c|c|c|c|c|}
\hline $\begin{array}{c}\text { Solanum aviculare G. } \\
\text { Forst. }\end{array}$ & fruit & $\begin{array}{l}\text { bush tomato, } \\
\text { kangaroo apple }\end{array}$ & $\begin{array}{c}\text { The fruit has a high antioxidant capacity } \\
\text { and was mainly used as a nutritious food. } \\
\text { The fruit has reported antibacterial and } \\
\text { anticancer properties. }\end{array}$ & unknown & $16,19,20$ \\
\hline $\begin{array}{c}\text { Tasmannia insipidia } \\
\text { R. Br. }\end{array}$ & leaves & brush pepperbush & $\begin{array}{c}\text { Used mainly as a flavouring agent. } \\
\text { No known traditional medicinal } \\
\text { uses however recent studies report } \\
\text { antibacterial activity }\end{array}$ & $\begin{array}{l}\text { phytochemistry of } \\
\text { other Tasmannia spp. } \\
\text { reports high levels of } \\
\text { terpenoids (especially } \\
\text { sesquiterpenoids). } \\
\text { Stilbenes are also } \\
\text { present. }\end{array}$ & $6,8,14,23$ \\
\hline $\begin{array}{l}\text { Tasmannia stipitata } \\
\text { (Vick.) A.C. Smith }\end{array}$ & leaves and berries & $\begin{array}{l}\text { Dorrigo pepper, } \\
\text { Northern } \\
\text { pepperbush }\end{array}$ & $\begin{array}{c}\text { Used mainly as a flavouring agent. } \\
\text { No known traditional medicinal } \\
\text { uses however recent studies report } \\
\text { antibacterial activity }\end{array}$ & $\begin{array}{l}\text { phytochemistry of } \\
\text { other Tasmannia spp. } \\
\text { reports high levels of } \\
\text { terpenoids (especially } \\
\text { sesquiterpenoids). } \\
\text { Stilbenes are also } \\
\text { present. }\end{array}$ & $6,8,14,23$ \\
\hline
\end{tabular}

Table 2: The mass of dried extracted material, the concentration after resuspension in deionised water, qualitative phytochemical screenings and antioxidant capacities of the Australian plant extracts.

\begin{tabular}{|c|c|c|c|c|c|c|c|c|c|c|c|c|c|c|c|}
\hline 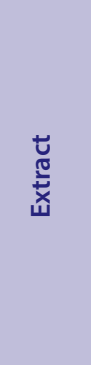 & 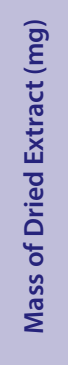 & 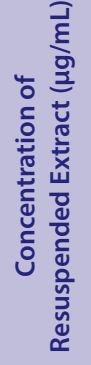 & 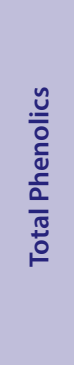 & 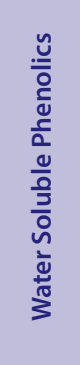 & 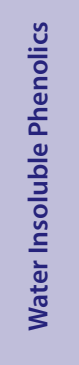 & 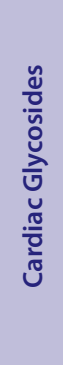 & 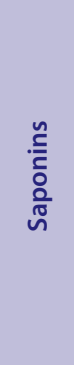 & 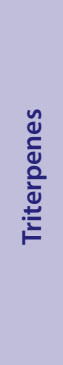 & 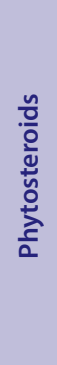 &  & 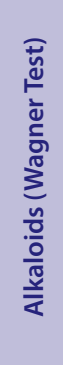 & $\begin{array}{l}\frac{n}{0} \\
\frac{0}{0} \\
\frac{0}{0} \\
\frac{\pi}{4}\end{array}$ & 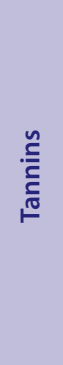 & 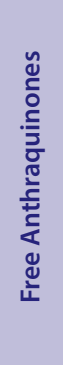 & 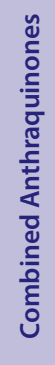 \\
\hline NTW & 52 & 5.2 & ++ & - & +++ & - & - & - & - & - & - & + & - & ++ & - \\
\hline NTM & 107 & 10.7 & +++ & - & +++ & - & ++ & - & - & - & - & + & - & - & - \\
\hline BTW & 79 & 7.9 & +++ & ++ & +++ & - & +++ & ++ & - & - & ++ & +++ & ++ & - & - \\
\hline BTM & 313 & 31.3 & +++ & - & +++ & - & - & ++ & - & - & ++ & ++ & - & - & - \\
\hline DLW & 182 & 18.2 & +++ & ++ & ++ & + & - & ++ & - & - & ++ & +++ & - & - & - \\
\hline DLM & 247 & 24.7 & + & - & - & + & ++ & ++ & - & - & - & +++ & - & - & - \\
\hline MW & 350 & 35 & +++ & +++ & +++ & - & +++ & ++ & - & - & - & +++ & ++ & - & - \\
\hline MM & 524 & 52.4 & +++ & +++ & - & - & +++ & ++ & - & - & - & +++ & ++ & - & - \\
\hline WSW & 120 & 12 & ++ & ++ & + & ++ & - & ++ & - & - & - & +++ & - & - & - \\
\hline WSM & 88 & 8.8 & ++ & +++ & ++ & - & ++ & ++ & - & - & ++ & +++ & - & - & - \\
\hline LAW & 162 & 16.2 & +++ & - & - & + & +++ & ++ & - & - & - & +++ & - & - & - \\
\hline LAM & 360 & 36 & +++ & - & - & + & - & ++ & - & - & - & +++ & - & - & - \\
\hline THW & 52 & 5.2 & +++ & +++ & +++ & ++ & +++ & ++ & - & - & ++ & +++ & ++ & ++ & - \\
\hline THM & 171 & 17.1 & +++ & +++ & ++ & ++ & +++ & ++ & - & - & ++ & +++ & ++ & - & - \\
\hline IPW & 195 & 19.5 & +++ & ++ & +++ & - & +++ & ++ & - & - & - & ++ & ++ & ++ & ++ \\
\hline IPM & 314 & 31.4 & +++ & +++ & +++ & - & +++ & ++ & - & - & - & ++ & ++ & + & ++ \\
\hline NSW & 25 & 2.5 & +++ & +++ & ++ & ++ & +++ & ++ & - & - & - & +++ & ++ & - & - \\
\hline NSM & 109 & 10.9 & +++ & +++ & +++ & ++ & +++ & ++ & - & - & + & ++ & ++ & - & - \\
\hline RMW & 30 & 3 & ++ & ++ & ++ & ++ & - & ++ & - & - & - & +++ & - & - & - \\
\hline RMM & 120 & 12 & +++ & +++ & +++ & ++ & +++ & ++ & - & - & ++ & +++ & ++ & + & - \\
\hline NBW & 108 & 10.8 & +++ & + & ++ & ++ & +++ & ++ & - & - & + & ++ & + & - & - \\
\hline NBM & 192 & 19.2 & +++ & +++ & +++ & - & +++ & ++ & - & - & ++ & +++ & ++ & - & - \\
\hline EBLW & 125 & 12.5 & +++ & +++ & + & - & + & - & - & - & - & ++ & + & - & - \\
\hline EBLM & 143 & 14.3 & +++ & +++ & + & - & + & - & - & - & - & + & ++ & - & - \\
\hline
\end{tabular}




\begin{tabular}{|c|c|c|c|c|c|c|c|c|c|c|c|c|c|c|c|}
\hline EMLW & 222 & 22.2 & +++ & +++ & + & - & + & - & - & - & - & +++ & + & - & - \\
\hline EMLM & 280 & 28 & +++ & +++ & + & - & + & - & - & - & - & ++ & ++ & - & - \\
\hline SSLW & 210 & 21 & +++ & ++ & - & - & + & - & - & - & - & ++ & +++ & - & - \\
\hline SSLM & 116 & 11.6 & +++ & ++ & - & - & + & - & - & + & - & ++ & ++ & - & - \\
\hline TILW & 184 & 18.4 & +++ & ++ & + & - & + & + & - & - & - & ++ & - & - & - \\
\hline TILM & 221 & 22.1 & +++ & ++ & + & - & ++ & + & - & - & - & ++ & - & - & - \\
\hline TSLW & 232 & 23.2 & +++ & +++ & +++ & - & ++ & + & - & - & - & +++ & - & - & - \\
\hline TSLM & 293 & 29.3 & +++ & +++ & ++ & - & +++ & + & - & - & - & +++ & - & - & - \\
\hline TSBW & 207 & 20.7 & +++ & +++ & +++ & - & ++ & + & - & - & - & +++ & - & - & - \\
\hline TSBM & 279 & 27.9 & +++ & +++ & +++ & - & ++ & + & - & - & - & +++ & - & - & - \\
\hline
\end{tabular}

+++ indicates a large response; ++ indicates a moderate response; + indicates a minor response; - indicates no response in the assay. NTW=aqueous native tamarind extract; NTM=methanolic native tamarind extract; BTW=aqueous bush tomato extract; BTM=methanolic bush tomato extract; DLW=aqueous desert lime extract; DLM=methanolic desert lime extract; $M W=$ aqueous muntries extract; $M M=$ methanolic muntries extract; WSW=aqueous wattle seed extract; WSM=methanolic wattle seed extract; LAW=aqueous lemon aspen extract; LAM=methanolic lemon aspen extract; THW=aqueous native thyme extract; THM=methanolic native thyme extract; IPW=aqueous Illawarra plum extract; IPM=methanolic Illawarra plum extract; NSW=aqueous native sage extract; NSM=methanolic native sage extract; RMW=aqueous river mint extract; RMM=methanolic river mint extract; NBW=aqueous native basil extract; NBM=methanolic native basil extract; EBLW=aqueous E. baileyana leaf extract; EBLM=methanolic E. baileyana leaf extract; EMLW=aqueous E. major leaf extract; EMLM=methanolic E. major leaf extract; SSLW=aqueous S. spinescens leaf extract; SSLM=methanolic S. spinescens leaf extract; TILW=aqueous T. insipidia leaf extract; TILM=methanolic T. insipidia leaf extract; TSLW=aqueous T. stipitata leaf extract; TSLM=methanolic T. stipitata leaf extract; TSBW=aqueous T. stipitata berry extract; TSBM=methanolic T. stipitata berry extract.

\section{RESULTS}

\section{Liquid extraction yields and qualitative phytochemical screening}

Extraction of $1 \mathrm{~g}$ of the native Australian plant materials with the solvents yielded dried plant extracts ranging from $25 \mathrm{mg}$ (aqueous native sage extract) to $524 \mathrm{mg}$ (methanolic muntries extract) (Table 2). Methanol was a better extractant than water, with substantially higher extraction for most plant materials. However, this trend was not observed in the wattle seed and S. spinescens extracts, which had substantially higher yields in the aqueous extracts compared to the methanolic extracts.

An extensive range of phytochemicals was detected inboth the methanolic and aqueous extracts for all plant species tested by qualitative phytochemical screening (Table 2). Both solvents typically extracted high levels of phenolics (both water soluble and water insoluble phenolics) for all plant materials. Additionally, all extracts generally contained high levels of flavonoids and moderate to high levels of saponins. Low to moderate levelsof triperenoids were present in most extracts, with the exception of the native thyme, Eucalyptus spp. and S. spinescens extracts. Similarly, cardiac glycosides, alkaloids and tannins were detected in some, but not all of the extracts. Furthermore, when these classes of compound were detected, they were generally only in low to moderate abundance. All extracts were generally devoid of detectable levels of phytosteroids and anthraquinones.

\section{Antimicrobial activity}

To assess the inhibitory activity of the crude plant extracts against $S$. pyogenes, $10 \mu \mathrm{L}$ aliquots of each extract were screened using a disc diffusion assay. The bacterial growth was inhibited by 24 of the 34 extracts tested ( 71\%) (Figure 1). The methanolic E. baileyana and E. major extracts were the most potent inhibitors of $S$. pyogenes growth (as judged by zones of inhibition), with inhibition zones of $12.3 \pm 0.6$ and $13.3 \pm 0.6$ $\mathrm{mm}$ respectively. This compares favourably with the ampicillin control, which had an inhibitory zone of $12.0 \pm 1.0 \mathrm{~mm}$. Whilst less potent than the corresponding methanolic extracts, the aqueous E. baileyana and $E$. major extracts were also good $S$. pyogenes growth inhibitors, with 10 and $8.3 \pm 0.3 \mathrm{~mm}$ inhibitory zones respectively.
The antimicrobial efficacy was further quantified by determining the MIC values (Table 3). Several extracts were potent inhibitors of $S$. pyogenes growth, with MIC values $<1000 \mu \mathrm{g} / \mathrm{mL}(<10 \mu \mathrm{g}$ infused into the disc). The Eucalyptus spp. extracts were particularly potent. Indeed, MIC values of 341 (3.4 $\mu \mathrm{g}$ infused into the disc) and $88 \mu \mathrm{g} / \mathrm{mL}(8.8 \mu \mathrm{g}$ infused into the disc) were determined for the aqueous and methanolic E. baileyana extracts respectively. Similarly, MIC values of $134(1.3 \mu \mathrm{g}$ infused into the disc) and $53 \mu \mathrm{g} / \mathrm{mL}$ (5.3 $\mu \mathrm{g}$ infused into the disc) were determined for the aqueous and methanolic E. major extracts respectively. This compares well with the ampicillin control, which was tested at $10 \mu \mathrm{g}$ infused into the disc. The methanolic wattle seed extract, aqueous and methanolic lemon aspen extracts, aqueous native thyme extract, methanolic river mint extract and the methanolic native basil extract were similarly potent growth inhibitors, with MIC values $\leq 1000 \mu \mathrm{g} / \mathrm{mL}$. MIC values indicative of moderate inhibitory activity (1000-5000 $\mu \mathrm{g} /$ $\mathrm{mL}$ ) were determined for many of the other extracts (methanolic native tamarind, bush tomato, desert lime, native thyme, native basil, T. stipitata leaf extracts, as well as the aqueous wattle seed, river mint, native basil, T. stipitata leaf extracts). All other extracts were either low potency S. pyogenes growth inhibitors (MIC $>5000 \mu \mathrm{g} / \mathrm{mL}$ ) or were devoid of inhibitory activity.

\section{Quantification of toxicity}

All extracts were initially screened at $2000 \mu \mathrm{g} / \mathrm{mL}$ in the assay (Figure 2). For comparison, the reference toxin potassium dichromate $(1000 \mu \mathrm{g} / \mathrm{mL})$ was also assessed in the bioassay. The potassium dichromate reference toxin was rapid in its onset, inducing nauplii death within the first $3 \mathrm{~h}$ of exposure, with $100 \%$ mortality evident in the subsequent $4-5 \mathrm{~h}$ (unpublished results). The majority of the Australian plant extracts also induced significant Artemia nauplii toxicity, with $\geq 50 \%$ mortality rates at $24 \mathrm{~h}$. Indeed, only the aqueous desert lime, muntries, native thyme, native sage, river mint and native basil extracts, as well as both S. spinescens extracts, induced $<50 \%$ mortality following $24 \mathrm{~h}$ exposure. Thus, only these extracts were deemed nontoxic, whilst all others were deemed toxic.

To further evaluate the effect of toxin concentration on the induction of mortality, the extracts were serially diluted in artificial seawater to testacross a range of concentrations in the Artemia nauplii bioassay. Table 
Table 3: Minimum inhibitory concentration $(\mu \mathrm{g} / \mathrm{mL})$ of the plant extracts against $S$. pyogenes and $\mathrm{LC}_{50}$ values $(\mu \mathrm{g} / \mathrm{mL})$ in the Artemia nauplii bioassay

\begin{tabular}{|c|c|c|}
\hline Extract & MIC & $\mathrm{LC}_{50}$ \\
\hline NTW & - & 1862 \\
\hline NTM & 1194 & 1595 \\
\hline BTW & 7900 & 5372 \\
\hline BTM & 2240 & 3467 \\
\hline DLW & $>10,000$ & 3875 \\
\hline DLM & 4200 & - \\
\hline MW & $>10,000$ & - \\
\hline MM & - & 1965 \\
\hline wsw & 2000 & 6254 \\
\hline WSM & 867 & 5763 \\
\hline LAW & 607 & 1872 \\
\hline LAM & 597 & 1500 \\
\hline THW & 1000 & - \\
\hline Тнм & 1891 & 3358 \\
\hline IPW & - & 1956 \\
\hline IPM & $>10,000$ & 1664 \\
\hline NSW & - & 1831 \\
\hline NSM & 1278 & 4015 \\
\hline RMW & 1300 & - \\
\hline RMM & 537 & 2658 \\
\hline NBW & 1256 & 2480 \\
\hline NBM & 627 & 7185 \\
\hline EBLW & 341 & 897 \\
\hline EBLM & 88 & 455 \\
\hline EMLW & 134 & 1146 \\
\hline EMLM & 53 & 793 \\
\hline SSLW & - & - \\
\hline SSLM & - & - \\
\hline TILW & - & 1687 \\
\hline TILM & - & 1463 \\
\hline TSLW & 1867 & 1487 \\
\hline TSLM & 1265 & 1813 \\
\hline TSBW & - & 1530 \\
\hline TSBM & - & - \\
\hline PD & ND & 186 \\
\hline SW & ND & - \\
\hline
\end{tabular}

Numbers indicate the mean MIC and LC50 values of triplicate determinations. - indicates no inhibition. $\mathrm{NTW}=$ aqueous native tamarind extract; NTM=methanolicnative tamarind extract; $\mathrm{BTW}=$ aqueous bush tomato extract; $\mathrm{BTM}=$ methanolic bush tomato extract; DLW=aqueous desert lime extract; DLM=methanolic desert lime extract; $M W=$ aqueous muntries extract; $M M=$ methanolic muntries extract; WSW=aqueous wattle seed extract; WSM=methanolic wattle seed extract; LAW=aqueous lemon aspen extract; LAM=methanolic lemon aspen extract; THW=aqueous native thyme extract; THM=methanolic native thyme extract; IPW=aqueous Illawarra plum extract; IPM=methanolic Illawarra plum extract; NSW=aqueous native sage extract; NSM=methanolic native sage extract; RMW=aqueous river mint extract; $\mathrm{RMM}=$ methanolic river mint extract; $\mathrm{NBW}=$ aqueous native basil extract; $\mathrm{NBM}=$ methanolic native basil extract; EBLW=aqueous E. baileyana leaf extract; EBLM=methanolic E. baileyana leaf extract; $\mathrm{EMLW}=$ aqueous $E$. major leaf extract; EMLM=methanolic $E$. major leaf extract; $S S L W=$ aqueous $S$. spinescens leaf extract; SSLM=methanolic S. spinescens leaf extract; TILW=aqueous T. insipidia leaf extract; TILM=methanolic T. insipidia leaf extract; TSLW=aqueous T. stipitata leaf extract; TSLM=methanolic T. stipitata leaf extract; TSBW=aqueous $T$. stipitata berry extract; TSBM=methanolic T. stipitata berry extract. $\mathrm{PD}=$ potassium dichromate control; $\mathrm{SW}=$ seawater control. $\mathrm{ND}=$ the indicated test was not performed. 


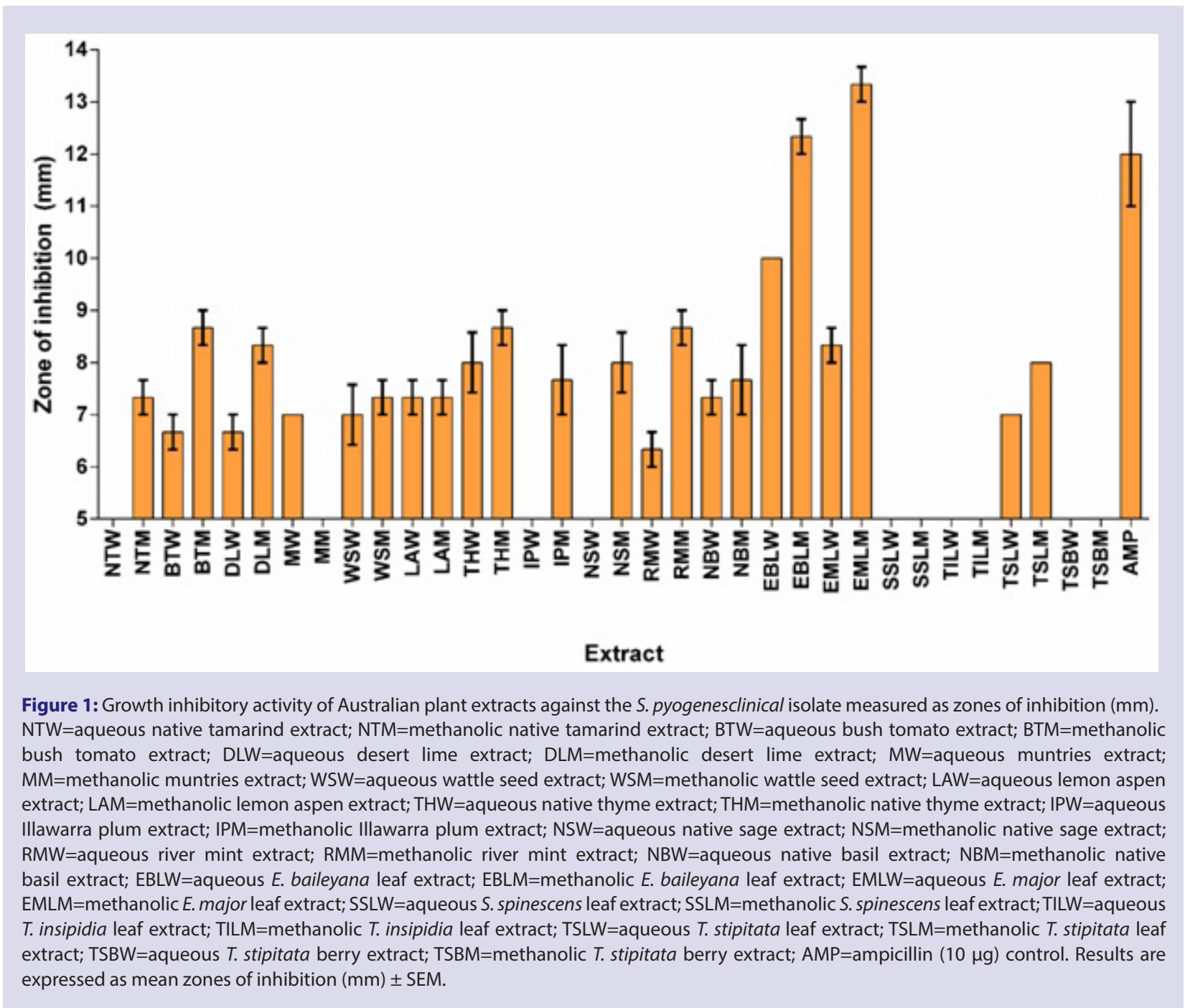

3 shows the $\mathrm{LC}_{50}$ values of the extracts towards $A$. franciscana. No $\mathrm{LC}_{50}$ values are reported for the aqueous desert lime, aqueous muntries, native thyme, native sage, river mint and native basil extracts, as well as both $S$. spinescens extractsas $<50 \%$ mortality was seen across all concentrations tested. With the exception of the aqueous E. major leaf extract, all Eucalyptus spp. extracts generally had $\mathrm{LC}_{50}$ values $<1000 \mu \mathrm{g} / \mathrm{mL}$. All other extracts yielded $\mathrm{LC}_{50}$ values substantially $>1000 \mu \mathrm{g} / \mathrm{mL}$ following $24 \mathrm{~h}$ exposure. As extracts with $\mathrm{LC}_{50}$ values of $>1000 \mu \mathrm{g} / \mathrm{mL}$ towards Artemia nauplii are deemed to be nontoxic, ${ }^{33}$ all extracts except the aqueous and methanolic E. baileyana extracts and the methanolic E. major leaf extract were deemed to be nontoxic.

\section{DISCUSSION}

Previous studies have reported potent bacterial growth inhibitory activity for all of the native Australian plant species screened in our study against a variety of pathogenic bacterial species. ${ }^{6,11-13,23}$ Extracts prepared from Australian fruits and culinary herbs, ${ }^{16,19,20}$ S. spinescens leaf extracts ${ }^{13}$ and Tasmannia spp. leaf and berry extracts ${ }^{6,8,23}$ have previously been reported to have inhibitory activity against extensive panels of pathogenic bacteria. With the exception of the S. spinescens, T. insipida and the T. stipatata berry extracts, all species screened in our study inhibited the growth of S. pyogenes. In contrast, the bacterial growth inhibitory properties of the
Eucalyptus spp. have been reported against a narrower range of pathogenic bacteria. ${ }^{11,12}$

The Eucalyptus spp. extracts displayed the most potent S. pyogenes growth inhibitory activity of the extracts tested in our study. Indeed, an MIC of $53 \mu \mathrm{g} / \mathrm{mL}$ was determined for the methanolic E. major leaf extract. However, despite being the most promising $S$. pyogenes growth inhibitory extracts, the methanolic and aqueous E. baileyana and methanolic E. major leaf extracts displayed substantial toxicity, with $\mathrm{LC}_{50}$ values as low as $455 \mu \mathrm{g} / \mathrm{mL}$ (methanolic E. baileyana leaf extract). Extracts with $\mathrm{LC}_{50}$ values $<1000 \mu \mathrm{g} / \mathrm{ml}$ towards Artemia nauplii are defined as being toxic, ${ }^{33}$ which may impact on their therapeutic potential. As the $\mathrm{LC}_{50}$ values are within the therapeutic ranges that would be required for $S$. pyogenes growth inhibition (determined by MIC), studies using human cell lines are required to further evaluate the safety of these extracts. However, even if the Eucalyptus spp. extracts are subsequently deemed unsafe for ingestion, they may still be useful $S$. pyogenes growth inhibitory agents. Impetigo is a cutaneous skin disease often resulting from $S$. pyogenes infection. ${ }^{34,35}$ Topical application of the extracts may prove effective in treating this form of the disease. Streptococcal pharyngitis is caused by Streptococcus spp. infectionson the pharynx surface. ${ }^{34}$ Therefore, gargling with solutions containing Eucalyptus spp. extracts may prove effective in treating this disease. However, streptococcal induced rheumatic fe- 




\section{Extract}

Figure 2: The lethality of the Australian plant extracts $(2000 \mu \mathrm{g} / \mathrm{mL})$ and the potassium dichromate $(1000 \mu \mathrm{g} / \mathrm{mL})$ and seawater controls towards Artemia franciscana nauplii after $24 \mathrm{~h}$ exposure.

NTW=aqueous native tamarind extract; NTM=methanolic native tamarind extract; BTW=aqueous bush tomato extract; BTM=methanolic bush tomato extract; $\mathrm{DLW}=$ aqueous desert lime extract; $\mathrm{DLM}=$ methanolic desert lime extract; $M W=$ aqueous muntries extract; $M M=$ methanolic muntries extract; $W S W=a q u e o u s$ wattle seed extract; $W S M=$ methanolic wattle seed extract; $L A W=a q u e o u s$ lemon aspen extract; $L A M=$ methanolic lemon aspen extract; $T H W=$ aqueous native thyme extract; $T H M=$ methanolic native thyme extract; IPW=aqueous Illawarra plum extract; IPM=methanolic Illawarra plum extract; NSW=aqueous native sage extract; NSM=methanolic native sage extract; $\mathrm{RMW}=$ aqueous river mint extract; $\mathrm{RMM}=$ methanolic river mint extract; $\mathrm{NBW}=$ aqueous native basil extract; $\mathrm{NBM=methanolic} \mathrm{native}$ basil extract; $\mathrm{EBLW}=$ aqueous $E$. baileyana leaf extract; $\mathrm{EBLM}=$ methanolic $E$. baileyana leaf extract; $\mathrm{EMLW}=$ aqueous $E$. major leaf extract; EMLM=methanolic $E$. major leaf extract; SSLW=aqueous $S$. spinescens leaf extract; SSLM=methanolic $S$. spinescens leaf extract; TILW=aqueous T. insipidia leaf extract; TILM=methanolic $T$. insipidia leaf extract; TSLW=aqueous $T$. stipitata leaf extract; TSLM=methanolic $T$. stipitata leaf extract; $\mathrm{TSBW}=$ aqueous $T$. stipitata berry extract; $\mathrm{TSBM}=$ methanolic $T$. stipitata berry extract; $\mathrm{AMP}=$ ampicillin $(10 \mu \mathrm{g})$ control. Results are expressed as mean zones of inhibition $(\mathrm{mm}) \pm \mathrm{SEM}$.

ver and rheumatic heart disease result from systemic infections, mainly affecting joint and cardiac tissue. Treatment of these diseases requires ingestion of antibiotics and anti-inflammatory drugs. The toxic nature of the Eucalyptus spp. extracts may preclude their use in the treatment of these diseases and instead limit them to topical applications.

Whilst an investigation of the phytochemistry of the Eucalyptus spp. extracts was beyond the scope of our study, plants of the genus Eucalyptus are well known for their high terpenoid contents. In particular, high 1, 8-cineole contents was reported for several Eucalyptus spp. ${ }^{5,10}$ Potent bacterial growth inhibitory activity has been reported for 1, 8-cineole against a panel of pathogenic bacteria. ${ }^{36}$ Another study reported MIC values for 1, 8-cineole against Staphylococcus aureus, Pseudomonas aeruginosa and Escherichia coli of between 16 and $256 \mu \mathrm{g} / \mathrm{mL} .{ }^{37}$ That study did not screen 1, 8-cineole against $S$. pyogenes. Eucalyptus spp. are also rich in a variety of other mono-and sesquiterpenoids. ${ }^{5,10}$ Some of these terpenoids have been previously reported to have potent broad spectrum antibacterial activity $^{14}$ and therefore may contribute to the $S$. pyogenes inhibitory activity.

Another commonality between the inhibitory Eucalyptus spp. extracts was that all contained relatively high levels of flavonoids and tannins. Many studies have reported potent growth inhibitory activities for a wide variety of flavonoids against extensive bacterial panels. ${ }^{38}$ Similarly, a number of tannin compounds have bacterial growth inhibitory activity. Gallotannins have been reported to inhibit the growth of a broad spectrum of bacterial species ${ }^{39}$ through a variety of mechanisms including binding cell surface molecules including lipotoichoic acid and proline-rich cell surface proteins, ${ }^{40,41}$ and by inhibiting glucosyltransferase enzymes. ${ }^{42}$ Elligitannins are also highly potent inhibitors of bacterial growth, with MIC values as low as $62.5 \mu \mathrm{g} / \mathrm{mL}^{39,41}$ Ellagitannins have also been reported to function via several antibiotic mechanisms including interaction with cytoplasmic oxidoreductases and by disrupting bacterial cell walls. ${ }^{39,41}$ Thus, it is likely that multiple compounds within the Eucalyptus spp. extracts are contributing to the growth inhibition of $S$. pyogenes.

Lemon aspen extracts, as well as the methanolic wattle seed, native basil and river mint extracts, were also potent $S$. pyogenes growth inhibitors, with MIC values substantially $<1000 \mu \mathrm{g} / \mathrm{mL}$. In contrast with the Eucalyptus spp. extracts, these extracts were nontoxic $\left(\mathrm{LC}_{50}\right.$ values substantially $>1000 \mu \mathrm{g} / \mathrm{mL}$ ). Thus, the therapeutic usage of these extracts need not be limited to external uses. Whilst the Eucalyptus spp. extracts may have greater efficacy in topical application, lemon aspen and wattle seed extracts would be more acceptable for systemic streptococcal induced rheumatic fever and rheumatic heart disease. A previous study has reported LC-MS evaluations of lemon aspen extracts prepared in the same 
way as those screened in our study. ${ }^{16} \mathrm{~A}$ number of interesting phytocompounds with antibacterial activity were detected. The identification of gingerol, rutin, luteolin, dihydrokaempferol, ellagic acid (and methylated ellagic acid derivatives) and chlorogenic acid was particularly noteworthy. Whether these compounds contribute to the $S$. pyogenes growth inhibitory activity reported here is yet to be determined.

The $S$. pyogenes growth inhibitory activity reported here is particularly noteworthy for the development of future antibiotic chemotherapeutics. Aside from the obvious antibiotic applications to directly treat localised throat (pharyngitis) and skin infections (impetigo), ${ }^{34,35}$ a number of substantially more serious illnesses are caused by acute and chronic $S$. pyogenes infections and may also benefit from treatment with these extracts. When $S$. pyogenes invades and colonises deeper tissue it can lead to erysipelas and cellulitis, conditions characterised by localised red, swollen and painful areas, and often by fever and lethargy. ${ }^{43-45}$ If not promptly treated, bacterium can spread to other areas via the bloodstream which may result in serious tissue damage and autoimmune diseases such as glomerulonephritis (inflammation of the glomeruli in the kidneys), lymphedema (inflammation of lymph nodes), septic arthritis and rheumatic fever (inflammation of cardiac tissue) ${ }^{44-46}$. Furthermore, acute $S$. pyogenes infections of subcutaneous tissues can induce the potentially fatal disease necrotizing fasciitis. ${ }^{46}$ These conditions are not only highly debilitating, but may also be life threatening and new, more effective treatment regimens could potentially prolong and increase the quality of life as well as reducing the burden on the health system.

\section{CONCLUSION}

The results of this study demonstrate the potential of Eucalyptus spp., lemon aspen, wattle seed, native basil and river mint extracts to block the growth of $S$. pyogenes. The toxicity of the Eucalyptus spp. extracts may limit their clinical usage to topical applications. However, the nontoxicity of the lemon aspen fruit, wattle seed, native sage and river mint extracts indicates their potential in the treatment of all manifestations of streptococcal disease, including systemic treatment. Further studies aimed at the purification of the bioactive components are needed to examine the mechanisms of action of these agents.

\section{ACKNOWLEDGEMENTS}

The authors are grateful to Michelle Mendell for the gift of the S. pyogenes clinical isolate used in these studies. Financial support for this work was provided by the Environmental Futures Research Institute and the School of Natural Sciences, Griffith University, Australia.

\section{CONFLICTS OF INTEREST}

The authors report no conflicts of interest.

\section{ABBREVIATIONS USED}

DMSO: Dimethyl sulfoxide; $\mathbf{L C}_{50}$ : The concentration required to achieve $50 \%$ mortality; MIC: Minimum inhibitory concentration.

\section{REFERENCES}

1. Chiller K, Selkin BA, Murakawa GJ. Skin Microflora and Bacterial Infections of the Skin. Journal of Investigative Dermatology Symposium Proceedings. $2001 ; 6(3): 170-4$

2. Hewitt WD, Farrar WE. Bacteremia and ecthyma caused by Streptococcus pyogenes in a patient with acquired immunodeficiency syndrome. Am J Med Sci. 1988;295(1):52-4

3. Dinkla K, Rohde M, Jansen WTM. Rheumatic fever-associated Streptococcus pyogenes isolates aggregate collagen. The Journal of Clinical Investigation. 2003;111(12):1905-12

4. Wright $\mathrm{MH}$, Sirdaarta J, Matthews B, et al. Growth inhibitory activity of kakadu Plum extracts against the opportunistic pathogen Clostridium perfringens: New leads in the prevention and treatment of clostridial myonecrosis. Pharmacognosy Journal. 2016b;8(2):144-53. DOI: 10.5530/pj.2016.2.8.

5. Cock IE. Medicinal and aromatic plants-Australia. In Ethnopharmacology, Encyclopedia of Life Support Systems (EOLSS), 2011. Developed under the auspices of UNESCO. Oxford, UK: EOLSS Publishers; 2011. Available from: http://www. eolss.net. Accessed 1 April 2013.

6. Hart C, Ilanko P, Sirdaarta J, et al. Tasmannia stipitata as a functional food/natural preservative: Antimicrobial activity and toxicity. Pharmacognosy Communications. 2014;4(4):33-47. DOI: 10.5530/pc.2014.4.4

7. Sautron C, Cock IE. Antimicrobial activity and toxicity of Syzygium australe and Syzygium leuhmanii fruit extracts. Pharmacognosy Communications. 2014;4(1):53-60

8. Winnett $\mathrm{V}$, Boyer $\mathrm{H}$, Sirdaarta J, et al. The potential of Tasmannia lanceolata as a natural preservative and medicinal agent: Antimicrobial activity and toxicity. Pharmacognosy Communications. 2014;4(1):42-52.

9. Cock IE, Mohanty S. Evaluation of the antibacterial activity and toxicity of Terminalia ferdinandia fruit extracts. Pharmacognosy Journal. 2011;3(20):72-9.

10. Lassak EV, McCarthyT. Australian medicinal plants. A complete guide to identification and usage. Reed New Holland Publishers 2011, Sydney Australia.

11. Cock IE. Antibacterial activity of selected Australian native plant extracts. Internet Journal of Microbiology. 2008;4(2):1-8.

12. Cock IE. Antimicrobial activity of Eucalyptus major and Eucalyptus baileyana methanolic extracts. Internet J Microbiol. 2009;6(1).

13. Cock IE, Kukkonen L. An examination of the medicinal potential of Scaevola spinescens: Toxicity, antibacterial and antiviral activities. Pharmacognosy Research. 2011;3(2):85-94. DOI: 10.4103/0974-8490.81955

14. Cock IE. The phytochemistry and chemotherapeutic potential of Tasmannia lanceolata (Tasmanian pepper): A review. Pharmacognosy Communications. 2013;3(4):13-25. DOI: 10.5530/pc.2013.4.3

15. Rayan P, Matthews B, McDonnell PA, et al. Phytochemical analysis of Tasmannia lanceolata extracts which inhibit Giardia duodenalis proliferation. Pharmacognosy Magazine 2015; in press.

16. Sirdaarta J, Maen A, Rayan $P$, et al. High performance liquid chromatographymass spectrometry analysis of high antioxidant Australian fruits with antiproliferative activity against cancer cells. Pharmacognosy Magazine 2016; in press.

17. Mohanty S, Cock IE. The chemotherapeutic potential of Terminalia ferdinandiana: Phytochemistry and Bioactivity. Pharmacog Rev. 2012;6(11):29-36.

18. Netzel M, Netzel G, Tian Q. Native Australian fruits-a novel source of antioxidants for food. Innov Food Sci Emerg Technol. 2007;8(3):339-46.

19. Maen A, Cock IE. Inhibitory activity of high antioxidant Australian native fruits against the bacterial triggers of selected autoimmune diseases. Pharmacognosy Communications. 2015;5(1):48-58.

20. Maen A, Cock IE. Inhibitory activity of Australian culinary herb extracts against the bacterial triggers of selected autoimmune diseases. Pharmacognosy Communications. 2015;5(2):130-9.

21. Wright M, Matthews B, Arnold MSJ, et al. The prevention of fish spoilage by high antioxidant Australian culinary plants: Shewanella putrefaciens growth inhibition. International Journal of Food Science and Technology. 2016; DOI: 10.1111/ijfs. 13026

22. Cock IE, Matthews B. Metabolomic profiling of antiviral Scaevola spinescens extracts by high resolution tandem mass spectrometry. Acta Horticulturae 2016; in press.

23. Cock IE, Winnett V, Sirdaarta J. The potential of selected Australian medicinal plants with anti-Proteus activity for the treatment and prevention of rheumatoid arthritis. Pharmacognosy Magazine. 2015;42(Supp 1):S190-S208. DOI: 10.4103/0973-1296.157734

24. Coutts $\mathrm{RH}$, Catterall CP. Identifying the plants of Toohey Forest. Ecos Educational Publishers 1980; Nambour, Australia.

25. Arkhipov A, Sirdaarta J, Rayan $P$, et al. An examination of the antibacterial, antifungal, anti-Giardial and anticancer properties of Kigelia africana fruit extracts. Pharmacognosy Communications. 2014;4(3):62-76. DOI: 10.5530/pc.2014.3.7

26. Kalt FR, Cock IE. Gas chromatography-mass spectroscopy analysis of bioactive Petalostigma extracts: Toxicity, antibacterial and antiviral activities. Pharmacog nosy Magazine. 2014;10(Suppl 1):S37-49. DOI: 10.4103/0973-1296.127338

27. Vesoul J, Cock IE. The potential of Bunya Nut as an antibacterial food agent. Pharmacognosy Communications. 2012;2(1):72-9. DOI: 10.5530/pc.2012.1.13

28. Courtney R, Sirdaarta J, Matthews B, et al. Tannin components and inhibitory activity of Kakadu plum leaf extracts against microbial triggers of autoimmune inflammatory diseases. Pharmacognosy Journal. 2015;7(1):18-31.

29. Cock IE. Antimicrobial activity of Acacia aulacocarpa and Acacia complanta methanolic extracts. Pharmacognosy Communications. 2012;2(1):66-71. DOI: 10.5530/pc.2012.1.12

30. Wright MH, Matthews B, Greene AC, et al. Growth inhibition of the zoonotic bacteria Bacillus anthracis by high antioxidant Australian plants: New leads for the prevention and treatment of anthrax. Pharmacognosy Communications. 2015;5(3):173-89.

31. Cock IE. Assessment of the toxicity of selected Australian native plant extracts using the Artemia franciscana nauplii bioassay. Internet Journal of Toxicology. 
$2008 ; 5(2)$

32. Ruebhart DR, Wikramasinghe WA, Cock IE. Protective efficacy of the antioxidants vitamin E and Trolox against Microcystis aeruginosa and microcystin-LR in Artemia franciscana nauplii. Journal of Toxicology and Environmental Health Part A. 2009;72(24):1567-75.

33. Cock IE, Ruebhart DR. Comparison of the brine shrimp nauplii bioassay and the ToxScreen-II test for the detection of toxicity associated with Aloe vera (Aloe barbadensis Miller) leaf extract. Pharmacognosy Research. 2009;1(2):98-101.

34. Bisno AL, Gerber MA, Gwaltney JM. Practice Guidelines for the Diagnosis and Management of Group A Streptococcal Pharyngitis. Clinical Infectious Diseases. 2002:35(2):113-25.

35. Worrall GJ. Acute sore throat. Canadian Family Physician. 2007;53(11):1961-2

36. Van Vuuren SF, Viljoen AM. Antimicrobial activity of limonene enantiomers and 1,8-cineol alone and in combination. Flavour and Fragrance Journal. 2007:22:540-4

37. Hendry ER, Worthington T, Conway BR. Antimicrobial efficacy of eucalyptus oil ana 1,8-cineol alone and in combination with chlorhexidine digluconate against microorganisms grown in planktonic and biofilm cultures. Journal of Antimicrobial Chemotherapy. 2009;64:1219-25.

38. Narayana KR, Reddy MS, Chaluvadi MR. Bioflavonoids classification, pharmacological, biochemical effects and therapeutic potential. Indian Journal of Pharmacology. 2001;33(1):2-16

39. Buzzini P, Arapitsas P, Goretti M. Antimicrobial activity of hydrolysable tannins Mini-Reviews in Medicinal Chemistry. 2008;8(12):1179-87.
40. Wolinsky LE, Sote EO. Isolation of natural plaque-inhibiting substances from 'Nigerian chewing sticks'. Caries Research. 1984;18:216-25.

41. Hogg SD, Embery G. Blood-group-reactive glycoprotein from human saliva interacts with lipoteichoic acid on the surface of Streptococcus sanguis cells. Archives in Oral Biology. 1982;27(3):261-8

42. Wu-Yuan CD, Chen CY, Wu RT. Gallotannins inhibit growth, water-soluble glucan synthesis, and aggregation of Streptococci mutans. Journal of Dental Research. 1988;67(1):51-5

43. Wertheim HFL, Nghia HDT, Taylor W. Streptococcus suis: An Emerging Human Pathogen. Clin Infect Dis. 2009;48(5):617-25.

44. O'Brien KL, Wolfson LJ, Watt JP. Burden of disease caused by Streptococcus pneumoniae in children younger than 5 years: global estimates. The Lancet 2009;374(9693):893-902.

45. Johansson L, Thulin P, Low DE. Getting under the skin: The Immunopathogenesis of Streptococcus pyogenes deep tissue infections. Clinical Infectious Diseases. 2010;51(1):58-65

46. Gerber MA, Baltimore RS, Eaton CB. Prevention of rheumatic fever and diag nosis and treatment of acute Streptococcal pharyngitis. A scientific statement from the American Heart Association Rheumatic Fever, Endocarditis, and Kawasaki Disease Committee of the Council on Cardiovascular Disease in the Young, the Interdisciplinary Council on Functional Genomics and Translationa Biology, and the Interdisciplinary Council on Quality of Care and Outcomes Research: Endorsed by the American Academy of Pediatrics. Circulation. $2009 ; 119(11): 1541-51$.
PICTORIAL ABSTRACT

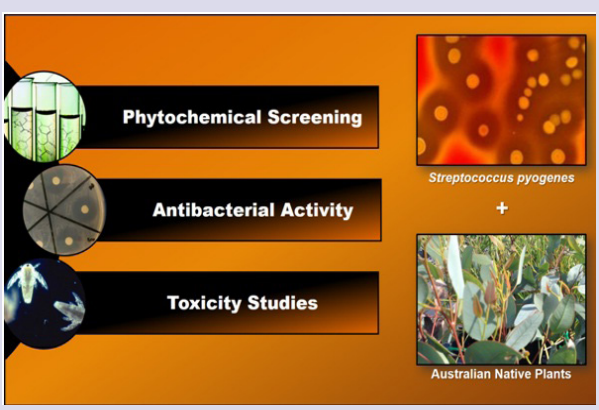

\section{SUMMARY}

- E. major extracts were particularly potent $S$. pyogenes growth inhibitors, with MIC values of 134 and $53 \mu \mathrm{g} / \mathrm{mL}$ respectively.

- Aqueous and methanolic E. baileyana extracts were also potent inhibitors of S. pyogenes growth with MIC values of 341 and $88 \mu \mathrm{g} / \mathrm{mL}$ respectively.

- The lemon aspen extracts as well as the methanolic wattle seed, native basil and river mint extracts were also potent growth inhibitors with MIC values substantially $<1000 \mu \mathrm{g} / \mathrm{mL}$

- All extracts except the Eucalyptus spp. Extracts were nontoxic in the Artemia nauplii assay.

\section{ABOUT AUTHORS}



Mr Cameron Lee: Completed his Bachelor of Science (BSc) in 2015 and is currently concluding his honours year. His research involves the investigation of thermophilic anaerobes that utilize toxic metals in anaerobic respiration (including uranium and arsenic). He has extensive experience in anaerobic cultivation/isolation and in numerous analytical techniques associated with heavy metal analysis.

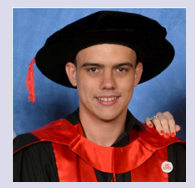

Dr Wright: Received his PhD in 2014, for his work investigating the manganese reduction and oxidation characteristics of environmental bacteria. He is currently a postdoctoral researcher at Griffith University, Australia, where he is working on several projects both in the areas of geomicrobiology and pharmacognosy. His present research interests are the use of bacteriogenic manganese oxides in the bioremediation of metal-contaminated sites as well as the use of Australian native plants in the treatment and prevention of various pathogenic bacteria.



Megan Arnold: Is currently undertaking her PhD in Tropical Parasitology at Griffith University's Eskitis Institute for Drug Discovery with a focus on the identification and development of novel chemoprophylactic agents for malaria. Her other research interests include investigating Australian high antioxidant plants for their antibacterial capabilities.

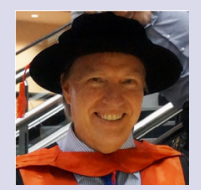

Dr Anthony Greene: Is a senior lecturer and researcher at Griffith University, Brisbane Australia. He obtained his $\mathrm{PhD}$ in Microbiology from the University of New South Wales and focuses on extreme environments, Bioremediation and Geomicrobiology. His specific interests include the microbial ecology of thermophilic, saline and alkaliphilic environments and the mechanisms and industrial potential of extremophilic bacteria contained therein.

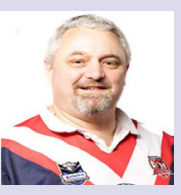

Dr lan Cock: Leads a research team in the Environmental Futures Research Institute and the School of Natural Sciences at Griffith University, Australia. His research involves bioactivity and phytochemical studies into a variety of plant species of both Australian and international origin, including Aloe vera, South Asian and South American tropical fruits, as well as Australia plants including Scaevola spinescens, Pittosporum phylliraeoides, Terminalia ferdinandiana (Kakadu plum), Australian Acacias, Syzygiums, Petalostigmas and Xanthorrhoea johnsonii (grass trees). This range of projects has resulted in nearly 200 publications in a variety of peer reviewed journals. 